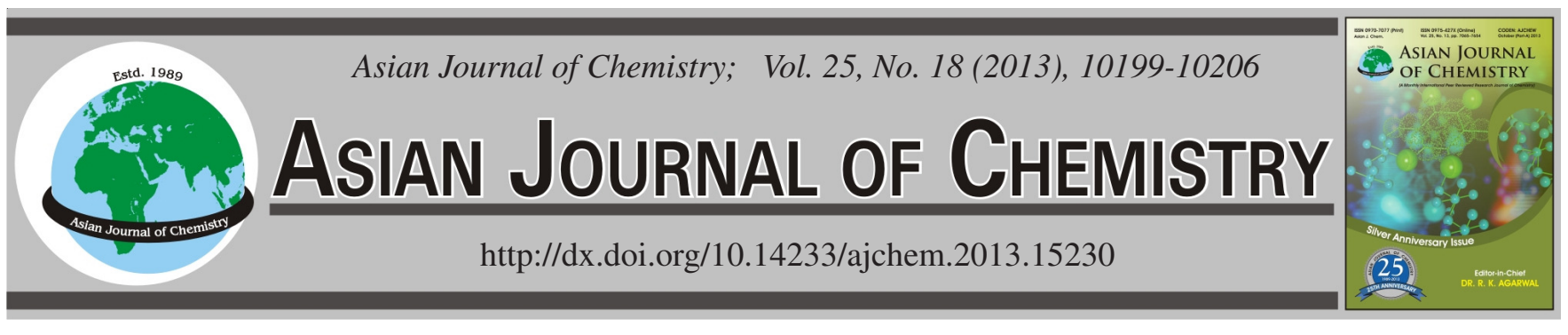

\title{
Synthesis of Novel 2,3-Disubstituted 1,4-Naphthoquinone Derivatives Containing Indole, Quinoline, Thiazole and Imidazole Moieties
}

\author{
Monirah A. Al-Alshaikh and Siham A. LahSasni"
}

Department of Chemistry, Faculty of Science, King Saud University, P.O. Box 22452, Riyadh 11495, Saudi Arabia

*Corresponding author: E-mail: slahsasni@ksu.edu.sa

\begin{abstract}
The present work describes one-pot multicomponent synthesis in which Michael addition-elimination reactions of the precursors 2-chloro-3-(2-arylhydrazinyl)naphthalene-1,4-dione, 2-amino-4,9-dioxo-4,9-dihydro- $1 \mathrm{H}$-benzo[f]indole-3-carbonitrile and 2-amino-4aryl-5,10-dioxo-5,10-dihydrobenzo[g]quinoline-3-carbonitrile with carbon disulphide, followed by intramolecular cyclization in the presence of pyridine or sulphuric acid or hydrazine have led to formation of the corresponding 3-arylamino-2-thioxo-2,3-dihydronaphtho[2,3- $d$ ] thiazole-4,9-dione, 2-thioxo-2H-benzo[f]thiazolo[4,5-b]indole-5-10(3H,4H)-dione, 2-mercaptobenzo[f]thiazolo[4,5b]indole-5-10 $(1 H, 4 H)$-dione, 11-aryl-2-thioxo-2,3-dihydrotobenzo[g]thiazolo[4,5-b]quinoline-5,10-dione and 1-amino-11-aryl-2mercapto-1 $H$-benzo[g]imidazo[4,5-b]quinoline-5,10-dione. The synthesized compounds have been identified and their structures are in confirmation with various spectroscopic techniques including IR, ${ }^{1} \mathrm{H}$ NMR, ${ }^{13} \mathrm{C}$ NMR and mass spectra.
\end{abstract}

Key Words: 2,3-Dichloro1,4-naphthoquinone, Michael addition-elimination, Intramolecular cyclization.

\section{INTRODUCTION}

The chemistry of quinone annulated heterocycles is highly dependent on the substituents at the quinonic or the adjacent rings $s^{1,2}$. Among various classes of heterocyclic quinones, naphthofluroquinones have attracted extensive interest owing to their presence in natural products and their versatile pharmacological activities. In this regard, many naphthofluroquinones are identified as natural products exhibiting a broad spectrum of biological activity ${ }^{3-6}$.

Furthermore, as a result of their redox properties, hetero1,4-naphthoquinones have shown potent biological affinity towards viral ${ }^{7}$, molluscidal $^{8}$, malarial $^{9}$, leishmanial $^{10}$, cancer $^{11}$, bacterial and fungal diseases ${ }^{12,13}$.

Structure-activity relationship studies on heterocyclic quinonoid compounds revealed that ring number and the position of the nitrogen or sulfur atoms in the heterocyclic ring play important role in manipulating the physiological and biological activities ${ }^{14-16}$ of these compounds.

Thus, in order to study the effect of different heterocyclic (e.g. indole, quinoline, thiazole and thione) quinonoid on the antibacterial and antifungal activities, we have carried out the synthesis, reactions and biological applications of a series of 2,3-disubstituted-1,4-naphthoquinone derivatives via utilization of 2,3-dichloro-1,4-naphthoquinone substrate in presence of the strong nucleophiles.

\section{EXPERIMENTAL}

All synthetic procedures were undertaken via Schlenk technique with dried solvents. Most reagents were purchased from Across, Sigma Aldrich and Merck. All synthesized compounds gave satisfactory elemental analysis for $\mathrm{C}, \mathrm{H}$ and $\mathrm{N}$. Flash chromatography was carried out using a glass column with silica gel (particle size 0.035-0.07 mm, pore diameter ca. $6 \mathrm{~nm}$ ). Solvent systems were determined via initial TLC analysis (Merck, silica gel 60F254).

Melting points were determined using an electrothermal's IA9000 series digital capillary melting point apparatus and used without correction. IR spectra were obtained, as $\mathrm{KBr}$ discs, a 1000-Perkin Elmer FT-IR spectrophotometer. Spectroscopic data were recorded as follows: ${ }^{1} \mathrm{H}$ and ${ }^{13} \mathrm{C}$ NMR spectra were acquired on a JEOL ECP-600 NMR in $\mathrm{CDCl}_{3}$ (or DMSO$d_{6}$ ) using TMS as an internal standard. Chemical shifts are given in $\delta \mathrm{ppm}$. Mass spectra were collected using a direct inlet system $(70 \mathrm{eV})$ with a VL detector (ES, $4000 \mathrm{~V})$.

Synthesis of 2-chloro-3-(4-arylhydrazinyl)naphthalene-1,4-dione: A mixture of 2,3-dichloro-1,4-naphthoquinone (1) (0.4 g, $0.88 \mathrm{mmol})$ and aryl hydrazine 2a-d $(0.88$ mmol) in ethanol (15 mL) was stirred in ice-bath for 1-4 h; the solid product was filtered off and washed with ethanol. Flash chromatography on silica gel using methanol/chloroform (1:4) as eluent gave 2-chloro-3-(2-arylhydrazinyl)- 
naphthalene-1,4-dione (3a-d) as a solid crystals with different colours as shown below.

2-Chloro-3-(2-phenylhydrazinyl)naphthalene-1,4dione (3a): Red needles, yield: (80 \%); m.p. 191-193 ${ }^{\circ} \mathrm{C}$; IR $\left(\mathrm{KBr}, \mathrm{v}_{\max }, \mathrm{cm}^{-1}\right): 3376$ and $3213(\mathrm{NH}), 1659$ and $1579(\mathrm{C}=\mathrm{O}$ of quinone); ${ }^{1} \mathrm{H}$ NMR (DMSO- $d_{6}$ ): $\delta$ ppm 3.65 (bs, $1 \mathrm{H}, \mathrm{NH}$ ), 7.33-7.46 (m, 3H, phenyl), 7.52-7.57 (m, 2H, Phenyl), 7.858.06 (m, 2H, C5-H and C8-H), 8.13-8.18 (m, 2H, C6-H and C7-H); 9.75 (bs, $1 \mathrm{H}, \mathrm{NH}) ;{ }^{13} \mathrm{C}$ NMR: $113.16,113.84,121.69$, $122.65,124.18,125.95,127.45,128.53,129.13,137.9,142.2$, 156.2 ( $s p^{2}$ carbons), 179.71 and $187.45(\mathrm{C}=\mathrm{O})$; Mass $\left(\mathrm{M}^{+}\right)$: 298.05; anal. calcd. (\%) for $\mathrm{C}_{16} \mathrm{H}_{11} \mathrm{~N}_{2} \mathrm{O}_{2} \mathrm{Cl}$ : C, 64.41; H, 3.69; N,9.39. Found (\%): C, 64.18; H, 3.68; N, 9.27; Beilstein test ${ }^{17}$ : $\mathrm{Cl}$ positive.

2-Chloro-3-[4-(4-nitrophenyl)hydrazinyl]naphthalene-1,4-dione (3b): Yellow needles, yield: (65\%); m.p. 220-123 ${ }^{\circ} \mathrm{C}$; IR (KBr, $\left.v_{\max }, \mathrm{cm}^{-1}\right): 3379$ and $3214(\mathrm{NH}), 1658$ and $1586\left(\mathrm{C}=\mathrm{O}\right.$ of quinone) $\mathrm{cm}^{-1} ;{ }^{1} \mathrm{H}$ NMR (DMSO- $\left.d_{6}\right): \delta$ ppm 3.65 (bs, 1H, NH), 6.92-7.03 (m, 2H, nitrophenyl), 7.85$8.06(\mathrm{~m}, 2 \mathrm{H}, \mathrm{C} 5-\mathrm{H}$ and $\mathrm{C} 8-\mathrm{H}), 8.12-8.17$ (m, 2H, C6-H and C7-H); 8.21-8.27 (m, 2H, nitrophenyl), 9.45 (bs, 1H, NH); ${ }^{13} \mathrm{C}$ NMR: $113.16,113.84,121.69,122.65,124.18,124.61$, $125.95,127.45,128.53,129.13,137.7,148.2,156.2\left(s p^{2}\right.$ carbons), 179.61 and $187.42(\mathrm{C}=\mathrm{O})$; mass $\left(\mathrm{M}^{+}\right): 343.81$; anal. calcd. (\%) for $\mathrm{C}_{16} \mathrm{H}_{10} \mathrm{~N}_{3} \mathrm{O}_{4} \mathrm{Cl}: \mathrm{C}, 55.84 ; \mathrm{H}, 2.90 ; \mathrm{N}, 12.24$. Found (\%): C, 55.53; H, 2.60; N, 12.07; Beilstein test ${ }^{17}: \mathrm{Cl}$ positive.

2-Chloro-3-(4-(4-chlorophenyl)hydrazinyl)naphthalene-1,4-dione(3c): Yellow needles, yield: (75\%); m.p. 205-207 ${ }^{\circ} \mathrm{C}$; IR (KBr, $\left.v_{\max }, \mathrm{cm}^{-1}\right)$ : 3381 and $3223(\mathrm{NH}), 1657$ and $1596\left(\mathrm{C}=\mathrm{O}\right.$ of quinone $\mathrm{cm}^{-1} ;{ }^{1} \mathrm{H}$ NMR (DMSO- $\left.d_{6}\right): \delta$ ppm 3.55 (bs, $1 \mathrm{H}, \mathrm{NH}$ ), 6.62-6.72 (m, 2H, chlorophenyl), 7.617.67 (m, 2H, chlorophenyl), 7.85-7.06 ( $\mathrm{m}, 2 \mathrm{H}, \mathrm{C5}-\mathrm{H}$ and $\mathrm{C} 8-$ $\mathrm{H}), 8.12-8.17$ (m, 2H, C6-H and C7-H); 9.55 (bs, 1H, NH); ${ }^{13} \mathrm{C}$ NMR: $113.16,113.84,121.69,122.65,124.18,124.61$, $125.95,127.45,128.53,129.13,136.8,140.2,156.2\left(s p^{2}\right.$ carbons), 179.61 and $187.42(\mathrm{C}=\mathrm{O})$; mass $\left(\mathrm{M}^{+}\right): 333.41$; anal. calcd. (\%) for $\mathrm{C}_{16} \mathrm{H}_{10} \mathrm{~N}_{2} \mathrm{O}_{2} \mathrm{Cl}_{2}: \mathrm{C}, 57.65 ; \mathrm{H}, 3.00 ; \mathrm{N}, 8.40$. Found (\%): C, 57.23; H, 2.98; N, 8.07; Beilstein test ${ }^{17}$ : $\mathrm{Cl}$ positive.

2-Chloro-3-(4-(4-methoxyphenyl)hydrazinyl)naphthalene-1,4-dione(3d): Greenish yellow needles, yield: (80 $\%)$; m.p. 198-202 ${ }^{\circ} \mathrm{C}$; IR ( $\left.\mathrm{KBr}, \mathrm{v}_{\max }, \mathrm{cm}^{-1}\right): 3481$ and 3323 (NH), 1658 and $1593\left(\mathrm{C}=\mathrm{O}\right.$ of quinone) $\mathrm{cm}^{-1} ;{ }^{1} \mathrm{H}$ NMR (DMSO-d $)$ : $\delta$ ppm 3.50 (bs, $1 \mathrm{H}, \mathrm{NH}), 3.67$ (s, $3 \mathrm{H}, \mathrm{OCH}_{3}$ ), 6.52-6.62 (m, 2H, methoxyphenyl), 6.91-7.07 (m, 2H, methoxyphenyl), 7.85-8.06 (m, 2H, C5-H and C8-H), 8.128.17 (m, 2H, C6-H and C7-H); 9.25 (bs, $1 \mathrm{H}, \mathrm{NH}) ;{ }^{13} \mathrm{C}$ NMR: $58\left(\mathrm{CH}_{3}\right), 113.16,113.84,118.53,119.13,121.69,122.65$, $124.18,124.61,125.95,127.45,134.8,150.2,156.2\left(s p^{2}\right.$ carbons), 179.11 and $187.62(\mathrm{C}=\mathrm{O})$; Mass $\left(\mathrm{M}^{+}\right): 329.55$; anal. calcd. (\%) for $\mathrm{C}_{17} \mathrm{H}_{13} \mathrm{~N}_{2} \mathrm{O}_{3} \mathrm{Cl}$ : C, 61.90; H, 3.95; N, 8.49. Found (\%): C, 61.79; H, 3.78; N, 8.38; Beilstein test ${ }^{17}: \mathrm{Cl}$ positive.

Synthesis of 3-arylamino-2-thioxo-2,3-dihydronaphtho[2,3-d] thiazole-4,9-dione: A mixture of 2-chloro-3(4-arylhydrazinyl)naphthalene-1,4-dione (3a-d) (0.01 mol) and carbon disulfide $(0.05 \mathrm{~mol})$ in pyridine $(10 \mathrm{~mL})$ was heated in oil-bath for 12-16 h. After cooling, ethanol was added and the precipitated solid was collected by filtration, purified via column chromatography on silica gel using ethyl acetate/hexane (1:4) as eluent to give 3-arylamino-2-thioxo-2,3-dihydro-naphtho[2,3-d]thiazole-4,9-dione $\mathbf{4 a - d}$ as yellow powders.

3-Phenylamino-2-thioxo-2,3-dihydro-naphtho[2,3d]thiazole-4,9-dione (4a): Yellow powder, yield: (60\%); m.p. 230-233 ${ }^{\circ} \mathrm{C}$; IR (KBr, $v_{\max }, \mathrm{cm}^{-1}$ ): $3423(\mathrm{NH}), 1656$ and 1586 (C=O of quinone), $1190(\mathrm{C}=\mathrm{S}$ of thiazole $) ;{ }^{1} \mathrm{H}$ NMR (DMSO$\left.d_{6}\right)$ : $\delta$ ppm 4.12 (bs, $\left.1 \mathrm{H}, \mathrm{NH}\right), 7.31-7.45$ (m, 3H, phenyl), 7.527.57 (m, 2H, phenyl), 7.85-8.06 (m, 2H, C5-H and C8-H), 8.13-8.18 (m, 2H, C6-H and C7-H) ${ }^{13} \mathrm{C}$ NMR: 113.16, 113.84, 114.09, 122.65, 124.18, 125.95, 127.45, 128.53, 129.13, 137.9, 142.2, 166.2 ( $s p^{2}$ carbons), 179.71 and $187.45(\mathrm{C}=\mathrm{O}), 190.12$ $(\mathrm{C}=\mathrm{S})$; mass $\left(\mathrm{M}^{+}\right): 338.47$; anal. calcd. $(\%)$ for $\mathrm{C}_{17} \mathrm{H}_{10} \mathrm{~N}_{2} \mathrm{O}_{2} \mathrm{~S}_{2}$ : C, 60.27; H, 2.96; N, 8.27. Found (\%): C, 60.08; H, 2.62; N, 7.99 .

3-(4-Nitrophenyl)amino-2-thioxo-2,3-dihydronaphtho[2,3-d]thiazole-4,9-dione (4b): Greenish yellow powder, yield: $(55 \%)$; m.p. $262-264{ }^{\circ} \mathrm{C}$; IR $\left(\mathrm{KBr}, \mathrm{v}_{\max }, \mathrm{cm}^{-1}\right)$ : $3479(\mathrm{NH}), 1658$ and $1586(\mathrm{C}=\mathrm{O}$ of quinone), $1196(\mathrm{C}=\mathrm{S}$ of thiazole); ${ }^{1} \mathrm{H}$ NMR (DMSO- $\left.d_{6}\right)$ : $\delta$ ppm: 4.65 (bs, $1 \mathrm{H}, \mathrm{NH}$ ), 6.92-7.03 (m, 2H, nitrophenyl), 7.85-8.06 (m, 2H, C5-H and C8-H), 8.12-8.17 (m, 2H, C6-H and C7-H); 8.21-8.27 (m, 2H, nitrophenyl), 9.45 (bs, $1 \mathrm{H}, \mathrm{NH}) ;{ }^{13} \mathrm{C}$ NMR: 113.16, 113.84 , $114.18,121.69,122.65,124.61,125.95,127.45,128.53$, $129.13,137.7,148.2,166.32$ ( $s p^{2}$ carbons), 179.61 and 187.42 $(\mathrm{C}=\mathrm{O})$; $190.12(\mathrm{C}=\mathrm{S})$; mass $\left(\mathrm{M}^{+}\right)$: 383.44; anal. calcd. $(\%)$ for $\mathrm{C}_{17} \mathrm{H}_{9} \mathrm{~N}_{3} \mathrm{O}_{4} \mathrm{~S}_{2}: \mathrm{C}, 53.20 ; \mathrm{H}, 2.35 ; \mathrm{N}, 10.97$. Found (\%): C, 52.98; $\mathrm{H}, 2.03$; N, 10.63 .

3-(4-Chlorophenyl)amino-2-thioxo-2,3-dihydronaphtho[2,3-d]thiazole-4,9-dione (4c): Greenish yellow powder, yield: $(57 \%)$; m.p. $250-253{ }^{\circ} \mathrm{C}$; IR $\left(\mathrm{KBr}, \mathrm{v}_{\max }, \mathrm{cm}^{-1}\right)$ : $3483(\mathrm{NH}), 1657$ and $1596\left(\mathrm{C}=\mathrm{O}\right.$ of quinone); ${ }^{1} \mathrm{H}$ NMR (DMSO- $\left.d_{6}\right)$ : $\delta$ ppm: 6.62-6.72 (m, 2H, chlorophenyl), 7.617.67 (m, 2H, chlorophenyl), 7.85-7.06 (m, 2H, C5-H and C8$\mathrm{H}), 8.12-8.17$ (m, 2H, C6-H and C7-H); 9.55 (bs, 1H, NH); ${ }^{13} \mathrm{C}$ NMR: $113.16,113.84,114.18,121.69,122.65,124.61$, $125.95,127.45,128.53,129.13,136.8,140.2,166.2\left(s p^{2}\right.$ carbons $), 179.61$ and $187.42(\mathrm{C}=\mathrm{O}) ; 190.10(\mathrm{C}=\mathrm{S})$; mass $\left(\mathrm{M}^{+}\right)$:373.34; anal. calcd. (\%) for $\mathrm{C}_{17} \mathrm{H}_{9} \mathrm{~N}_{2} \mathrm{O}_{2} \mathrm{~S}_{2} \mathrm{Cl}$ : C, 54.70; H, 2.41; N, 7.50. Found (\%): C, 54.45; H, 2.23; N, 7.29.

3-(4-Methoxyphenyl)amino-2-thioxo-2,3-dihydronaphtho[2,3-d]thiazole-4,9-dione (4d): Greenish yellow powder, yield: $(68 \%)$; m.p. $239-242{ }^{\circ} \mathrm{C}$; IR $\left(\mathrm{KBr}, v_{\max }, \mathrm{cm}^{-1}\right)$ : $3483(\mathrm{NH}), 1657$ and $1596\left(\mathrm{C}=\mathrm{O}\right.$ of quinone); ${ }^{1} \mathrm{H}$ NMR (DMSO- $\left.d_{6}\right): \delta$ ppm: $3.67\left(\mathrm{~s}, 3 \mathrm{H}, \mathrm{OCH}_{3}\right), 6.52-6.62(\mathrm{~m}, 2 \mathrm{H}$, methoxyphenyl), 6.91-7.07 ( $\mathrm{m}, 2 \mathrm{H}$, methoxyphenyl), 7.858.06 (m, 2H, C5-H and C8-H), 8.12-8.17 (m, 2H, C6-H and C7-H); 9.25 (bs, 1H, NH); ${ }^{13} \mathrm{C}$ NMR: $58\left(\mathrm{CH}_{3}\right), 113.16,113.84$, $114.18,118.53,119.13,121.69$, 122.65, 124.61, 125.95, $127.45,134.8,150.2,166.2$ ( $s p^{2}$ carbons), 179.11 and 187.62 $(\mathrm{C}=\mathrm{O}), 190.10(\mathrm{C}=\mathrm{S})$; mass $\left(\mathrm{M}^{+}\right)$: 369.49; anal. calcd. $(\%)$ for $\mathrm{C}_{18} \mathrm{H}_{12} \mathrm{~N}_{2} \mathrm{O}_{3} \mathrm{~S}_{2}: \mathrm{C}, 58.54 ; \mathrm{H}, 3.25 ; \mathrm{N}, 7.58$. Found (\%): C, 58.38; H, 3.06; N, 7.32.

Synthesis of 2-amino-4,9-dioxo-4,9-dihydro-1Hbenzo[f]indole-3-carbonitrile (5): A mixture of 2,3-dichloro1,4-naphthoquinone (1) (0.8 $\mathrm{mmol})$ and malonitrile $(0.8 \mathrm{mmol})$ in absolute ethanol was heated under reflux for $5 \mathrm{~h}$ in the presence of ammonium acetate $(4 \mathrm{mmol})$. The reaction mixture was then cooled, filtered off, then washed with absolute ethanol and air dried. Recrystallization from chloroform afforded 
2-amino-4,9-dioxo-4,9-dihydro- $1 H$-benzo[f]indole-3carbonitrile (5) as a violet powder. Violet powder, yield: (85 $\%$ ); m.p. $>300{ }^{\circ} \mathrm{C}$; IR $\left(\mathrm{KBr}, v_{\max }, \mathrm{cm}^{-1}\right): 3270(\mathrm{NH}), 3155$ and $3033\left(\mathrm{NH}_{2}\right), 2169(\mathrm{CN}), 1672$ and $1595(\mathrm{C}=\mathrm{O}$ of quinone $)$ $\mathrm{cm}^{-1} ;{ }^{1} \mathrm{H}$ NMR (DMSO- $d_{6}$ ): $\delta$ ppm: 2.49 (bs, $\mathrm{NH}_{2}$ ), 6.52 (bs, $\mathrm{NH}), 7-7.20(\mathrm{~m}, 1 \mathrm{H}, \mathrm{C} 5-\mathrm{H}), 7.64-7.76(\mathrm{~m}, 2 \mathrm{H}, \mathrm{C} 6-\mathrm{H}$ and $\mathrm{C} 7-$ $\mathrm{H})$; 7.89-7.91 (m, 1H, C8-H); ${ }^{13} \mathrm{C}$ NMR: $113.16(\mathrm{CN}), 121.40$, $125.34,126.27,131.43,131.85,132.83,134.26,144.35\left(s p^{2}\right.$ carbons), 171.97 and $182.645(\mathrm{C}=\mathrm{O})$; mass $\left(\mathrm{M}^{+}\right)$: 237.39; anal. calcd. (\%) for $\mathrm{C}_{13} \mathrm{H}_{7} \mathrm{~N}_{3} \mathrm{O}_{2}$ : C, 65.82; $\mathrm{H}, 2.95 ; \mathrm{N}, 17.72$. Found (\%): C, 65.68; H, 2.64; N, 17.58.

Synthesis of potassium 3-cyano-4,9-dioxo-4,9-dihydro$1 H$-benzo[f]indol-2-ylcarbamodithioate (6): A mixture of compound 5 (0.4 mmol) and carbon disulphide $(0.44 \mathrm{mmol})$ together with potassium hydroxide $(0.4 \mathrm{mmol})$ in absolute ethanol was stirred for $24 \mathrm{~h}$ at room temperature. Dry ether was then added and the precipitated solid was collected by filtration, thereby obtaining the corresponding potassium 3-cyano-4,9-dioxo-4,9-dihydro- $1 \mathrm{H}$-benzo[f]indol-2-ylcarbamodithioate $(\mathbf{6})$.

Synthesis of 2-thioxo-2H-benzo[f]thiazolo[4,5b]indole-5-10 $(3 H, 4 H)$-dione (7): An aqueous solution of potassium 3-cyano-4,9-dioxo-4,9-dihydro- $1 H$-benzo[f]indol2-yldithiocarbazate $(\mathbf{6})(0.04 \mathrm{mmol})$ was added dropwise with constant stirring to concentrated sulphuric acid $(98 \%, 15 \mathrm{~mL})$ and the reaction mixture was stirred for $24 \mathrm{~h}$. The mixture was cautiously added to crushed ice, stirred for $1 \mathrm{~h}$, refrigerated for $2 \mathrm{~h}$ and the precipitated solid was filtered off and purified by column chromatography using ethyl acetate/hexane (1:4) as eluent to give a green solid of the title compound 2-thioxo$2 \mathrm{H}$-benzo[f]thiazolo[4,5-b]indole-5-10(3H,4H)-dione (7). Green powder, yield: $(60 \%)$; m.p. $>300{ }^{\circ} \mathrm{C}$; IR $\left(\mathrm{KBr}, \mathrm{v}_{\max }\right.$, $\left.\mathrm{cm}^{-1}\right): 3428(\mathrm{NH}), 1656$ and $1586(\mathrm{C}=\mathrm{O}$ of quinone $) ;{ }^{1} \mathrm{H} \mathrm{NMR}$ (DMSO- $d_{6}$ ): $\delta$ ppm: 4.43 (bs, NH), 7-7.20 (m, 1H, C6-H), 7.64-7.76 (m, 2H, C7-H and C8-H); 7.89-7.91 (m, 1H, C9$\mathrm{H}), 9.85$ (bs, $1 \mathrm{H}, \mathrm{NH}) ;{ }^{13} \mathrm{C}$ NMR: $121.40,125.34,126.27$, $131.43,131.85,132.83,134.26,164.35$ ( $s p^{2}$ carbons), 171.97 and $182.645(\mathrm{C}=\mathrm{O}), 184.52(\mathrm{C}=\mathrm{S})$; mass $\left(\mathrm{M}^{+}\right)$: 286.56; anal. calcd. (\%) for $\mathrm{C}_{13} \mathrm{H}_{6} \mathrm{~N}_{2} \mathrm{O}_{2} \mathrm{~S}_{2}$ : C, 54.54; $\mathrm{H}, 2.09 ; \mathrm{N}, 9.79$. Found (\%): C, 54.28; H, 1.98; N, 9.54.

Synthesis of 2-mercaptobenzo[f]thiazolo[4,5-b]indole5-10 $(\mathbf{1 H}, \mathbf{4 H})$-dione $(\mathbf{8})$ : A mixture of compound 5 (0.04 $\mathrm{mmol})$ and $98 \%$ hydrazine hydrate $(10 \mathrm{~mL})$ was heated under reflux for $3 \mathrm{~h}$. After cooling, water was added and the mixture was neutralized with $10 \%$ hydrochloric acid. The separated crude product was then filtered off and purified via column chromatography using methanol/chloroform (1:4) as eluent to yield compound $\mathbf{8}$ in $25 \%$. Gray powder, yield: (55\%); m.p. $>300{ }^{\circ} \mathrm{C}$; IR $\left(\mathrm{KBr}, v_{\max }, \mathrm{cm}^{-1}\right): 3328(\mathrm{NH}), 3155$ and $3033\left(\mathrm{NH}_{2}\right), 2560(\mathrm{SH}), 1656$ and $1586(\mathrm{C}=\mathrm{O}$ of quinone); ${ }^{1} \mathrm{H}$ NMR (DMSO- $d_{6}$ ): $\delta$ ppm: 4.43 (bs, NH), 6.66 (bs, 2H, $\mathrm{NH}_{2}$ ), 7-7.20 (m, 1H, C6-H), 7.64-7.76 (m, 2H, C7-H and C8$\mathrm{H})$; 7.89-7.91 (m, 1H, C9-H), $12.05(\mathrm{~s}, 1 \mathrm{H}, \mathrm{SH}) ;{ }^{13} \mathrm{C}$ NMR: $121.40,125.34,126.27,131.43,131.85,132.83,134.26$, 164.35 ( $s p^{2}$ carbons), 171.97 and $182.645(\mathrm{C}=\mathrm{O})$; mass $\left(\mathrm{M}^{+}\right)$: 284.81; anal. calcd. (\%) for $\mathrm{C}_{13} \mathrm{H}_{8} \mathrm{~N}_{4} \mathrm{O}_{2} \mathrm{~S}$ : C, 54.93; H, 2.82; N, 19.72. Found (\%): C, 54.75; H, 2.56; N, 19.53 .

2-Arylidenemalononitrile (9a-d): All these compounds were prepared as reported ${ }^{23-25}$.
Synthesis of 2-amino-4-aryl-5,10-dioxo-5,10-dihydrobenzo[g]quinoline-3-carbonitrile: A mixture of 2,3-dichloro1,4-naphthoquinone $(0.3 \mathrm{mmol})$ 1, 2-arylidenemalononitrile (9a-d) $(0.3 \mathrm{mmol})$ and ammonium acetate $(1.2 \mathrm{mmol})$ in ethanol $(15 \mathrm{~mL})$ was heated under reflux for $4-7 \mathrm{~h}$. The solid product was filtered, washed with ethanol, dried and recrystallized from chloroform to yield compounds $\mathbf{1 0 a - d}$ as violet powders.

2-Amino-5,10-dioxo-4-phenyl-5,10-dihydrobenzo[g]quinoline-3-carbonitrile (10a): Yield: $(86 \%)$; m. p. > $300{ }^{\circ} \mathrm{C}$; IR $\left(\mathrm{KBr}, \mathrm{v}_{\max }, \mathrm{cm}^{-1}\right): 3250$ and $3138\left(\mathrm{NH}_{2}\right), 2171(\mathrm{CN}), 1676$ and $1586\left(\mathrm{C}=\mathrm{O}\right.$ of quinone); ${ }^{1} \mathrm{H}$ NMR (DMSO- $\left.d_{6}\right): \delta$ ppm: 5.51 (bs, $\left.2 \mathrm{H}, \mathrm{NH}_{2}\right), 7.09-7.18(\mathrm{~m}, 1 \mathrm{H}$, phenyl), 7.27-7.35 (m, 2H, phenyl), 7.46-7.52 (m, 2H, phenyl), 7.62-7.67 (m, 1H, C6-H), 7.72-7.77 (m, 1H, C9-H); 7.87-7.91 (m, 2H, C7-H and C8-H); ${ }^{13}$ C NMR: 112.40 (CN), 93.6, 120.59, 124.53, 125.45, 128.4, 130.64, 131.03, 132.04, 133.45, 143.60, 153, 156.3, 164.60 ( $s p^{2}$ carbons), 171.17 and $181.85(\mathrm{C}=\mathrm{O})$; Mass $\left(\mathrm{M}^{+}\right)$: 325.35; anal. calcd. (\%) for $\mathrm{C}_{20} \mathrm{H}_{11} \mathrm{~N}_{3} \mathrm{O}_{2}$ : C, 73.84; H, 3.38; N, 12.92. Found (\%): C, 73.55; H, 3.18; N, 12.73 .

2-Amino-5,10-dioxo-4-(4-nitrophenyl)-5,10-dihydrobenzo-[g]quinoline-3-carbonitrile (10b): Yield: (80\%); m.p. $>300{ }^{\circ} \mathrm{C}$; IR $\left(\mathrm{KBr}, \mathrm{v}_{\max }, \mathrm{cm}^{-1}\right): 3245$ and $3123\left(\mathrm{NH}_{2}\right), 2168$ $(\mathrm{CN}), 1676$ and $1586\left(\mathrm{C}=\mathrm{O}\right.$ of quinone); ${ }^{1} \mathrm{H}$ NMR (DMSO$\left.d_{6}\right)$ : $\delta$ ppm: 5.51 (bs, $2 \mathrm{H}, \mathrm{NH}_{2}$ ), 7.56-7.60 (m, 2H, nitrophenyl), 7.62-7.67 (m, 1H, C6-H), 7.72-7.77 (m, 1H, C9-H); 7.87-7.91 (m, 2H, C7-H and C8-H), 8.27-8.35 (m, 2H, nitrophenyl); ${ }^{13} \mathrm{C}$ NMR: $112.40(\mathrm{CN}), 93.6,120.59,124.53,125.45,128.4$, 130.64, 131.03, 132.04, 140.45, 148.4, 153.0, 156.3, 164.60 ( $s p^{2}$ carbons), 171.17 and $181.85(\mathrm{C}=\mathrm{O})$; mass $\left(\mathrm{M}^{+}\right): 370.25$; anal. calcd. (\%) for $\mathrm{C}_{20} \mathrm{H}_{10} \mathrm{~N}_{4} \mathrm{O}_{4}: \mathrm{C}, 64.86 ; \mathrm{H}, 2.70 ; \mathrm{N}, 20.74$. Found (\%): C, 64.52; H, 2.47; N, 20.51.

2-Amino-5,10-dioxo-4-(4-chlorophenyl)-5,10-dihydrobenzo[g]quinoline-3-carbonitrile (10c): Yield: (84\%); m.p. $>300{ }^{\circ} \mathrm{C}$; IR ( $\left.\mathrm{KBr}, v_{\max }, \mathrm{cm}^{-1}\right): 3255$ and $3133\left(\mathrm{NH}_{2}\right), 2170$ $(\mathrm{CN}), 1676$ and $1586\left(\mathrm{C}=\mathrm{O}\right.$ of quinone); ${ }^{1} \mathrm{H}$ NMR (DMSO$\left.d_{6}\right): \delta$ ppm: 5.51 (bs, $\left.2 \mathrm{H}, \mathrm{NH}_{2}\right), 7.29-7.35$ (m, 2H, chlorophenyl), 7.53-7.58 (m, 2H, chlorophenyl), 7.62-7.67 (m, 1H, C6-H), 7.72-7.77 (m, 1H, C9-H); 7.87-7.91 (m, 2H, C7-H and C8-H); ${ }^{13}$ C NMR: 112.40 (CN), 93.6, 120.59, 124.53, 125.45, 128.4, 130.64, 131.03, 132.04, 134.4, 136.65, 153.0, 156.3, 164.60 ( $s p^{2}$ carbons), 171.17 and $181.85(\mathrm{C}=\mathrm{O})$; mass $\left(\mathrm{M}^{+}\right)$: 359.85; anal. calcd. (\%) for $\mathrm{C}_{20} \mathrm{H}_{10} \mathrm{~N}_{3} \mathrm{O}_{2} \mathrm{Cl}$ : C, 66.85; $\mathrm{H}, 2.78$; N, 11.70. Found (\%): C, 66.65; H, 2.43; N, 11.47 .

2-Amino-5,10-dioxo-4-(4-methoxyphenyl)-5,10dihydrobenzo[g]quinoline-3-carbonitrile (10d): Yield: (87 $\%)$; m.p. $>300^{\circ} \mathrm{C}$; IR $\left(\mathrm{KBr}, \mathrm{v}_{\max }, \mathrm{cm}^{-1}\right)$ : 3233 and $3123\left(\mathrm{NH}_{2}\right)$, $2170(\mathrm{CN}), 1676$ and $1586\left(\mathrm{C}=\mathrm{O}\right.$ of quinone); ${ }^{1} \mathrm{H}$ NMR (DMSO- $d_{6}$ ): $\delta$ ppm: 3.63 (s, $3 \mathrm{H}, \mathrm{CH}_{3}$ ), 5.51 (bs, $2 \mathrm{H}, \mathrm{NH}_{2}$ ), 7.09-7.15 (m, 2H, methoxyphenyl), 7.43-7.48 (m, 2H, methoxyphenyl), 7.62-7.67 (m, 1H, C6-H), 7.72-7.77 (m, 1H, C9-H); 7.87-7.91 (m, 2H, C7-H and C8-H); ${ }^{13} \mathrm{C}$ NMR: 56.8 $\left(\mathrm{CH}_{3}\right), 112.40(\mathrm{CN}), 93.6,114.4,120.59,124.53,125.45$, 130.64, 130.95, 131.03, 132.04, 153.0, 156.3, 160.14, 164.60 ( $s p^{2}$ carbons), 171.17 and $181.85(\mathrm{C}=\mathrm{O})$; mass $\left(\mathrm{M}^{+}\right)$: 355.85; anal. calcd. (\%) for $\mathrm{C}_{21} \mathrm{H}_{13} \mathrm{~N}_{3} \mathrm{O}_{3}: \mathrm{C}, 70.98 ; \mathrm{H}, 3.66 ; \mathrm{N}, 11.83$. Found (\%): C, 70.75; H, 3.48; N, 11.59 .

Synthesis of potassium 4-aryl-3-cyano-5,10-dioxo-5,10dihydrobenzo[g]quinolin-2-ylcarbamodithiate (11a-d): A mixture of compound 10a-d $(0.4 \mathrm{mmol})$ and carbon disulphide 
$(0.44 \mathrm{mmol})$ together with potassium hydroxide $(0.4 \mathrm{mmol})$ in absolute ethanol was stirred for $24 \mathrm{~h}$ at room temperature. Dry diethyl ether was then added and the precipitated solid was collected by filtration to give the corresponding potassium 4-aryl-3-cyano-5,10-dioxo-5,10-dihydrobenzo[ $g$ ] quinolin-2ylcarbamodithiate (11a-d).

Synthesis of 11-aryl-2-thioxo-2,3-dihydrotobenzo[g] thiazolo[4,5-b]quinoline-5,10-dione: A solution of potassium 4-aryl-3-cyano-5,10-dioxo-5,10-dihydrobenzo[ $g$ ]quinolin-2ylcarbamodithiate (11a-d) $(0.04 \mathrm{mmol})$ was added dropwise to a conc. sulphuric acid $(15 \mathrm{~mL}, 98 \%)$ and then stirred for 24 h. The mixture was cautiously added to crushed ice, stirred for $1 \mathrm{~h}$, refrigerated for $2 \mathrm{~h}$ and the separated precipitate was filtered off and purified by column chromatography using ethyl acetate/hexane (1:4) as eluent to afford 11-aryl-2-thioxo-2,3dihydrotobenzo[ $g]$ thiazolo[4,5-b]quinoline-5,10-dione.

11-Phenyl-2-thioxo-2,3-dihydrotobenzo[g]thiazolo[4,5-b]quinoline-5,10-dione (12a): Green powder, yield: (55 $\%$ ); m.p. $>300{ }^{\circ} \mathrm{C}$; IR (KBr, $\left.v_{\max }, \mathrm{cm}^{-1}\right): 3350(\mathrm{NH}), 1676$ and 1586 (C=O of quinone); ${ }^{1} \mathrm{H}$ NMR (DMSO- $\left.d_{6}\right): \delta$ ppm: 4.49 (bs, H, NH), 7.09-7.18 (m, 1H, phenyl), 7.27-7.35 (m, 2H, phenyl), 7.46-7.52 (m, 2H, phenyl), 7.62-7.67 (m, 1H, C6$\mathrm{H})$, 7.72-7.77 (m, 1H, C9-H); 7.87-7.91 (m, 2H, C7-H and $\mathrm{C} 8-\mathrm{H}) ;{ }^{13} \mathrm{C}$ NMR: $119.40,120.59,124.53,125.45,128.4$, $130.64,131.03,132.04,133.45,143.60,148.7,150.6,161.60$ ( $s p^{2}$ carbons), 171.17 and $181.85(\mathrm{C}=\mathrm{O}), 185.41(\mathrm{C}=\mathrm{S})$; mass $\left(\mathrm{M}^{+}\right)$: 374.40; anal. calcd. (\%) for $\mathrm{C}_{20} \mathrm{H}_{10} \mathrm{~N}_{2} \mathrm{O}_{2} \mathrm{~S}_{2}: \mathrm{C}, 64.17 ; \mathrm{H}$, $2.67 ; \mathrm{N}, 7.48$. Found (\%): C, 63.94; H, 2.38; N, 7.27.

11-(4-Nitrophenyl)-2-thioxo-2,3-dihydrotobenzo[g]thiazolo[4,5-b]quinoline-5,10-dione (12b): Green powder, yield: $(50 \%)$; m.p. $>300{ }^{\circ} \mathrm{C}$; IR $\left(\mathrm{KBr}, \mathrm{v}_{\max }, \mathrm{cm}^{-1}\right): 3345(\mathrm{NH})$, 1676 and $1586\left(\mathrm{C}=\mathrm{O}\right.$ of quinone); ${ }^{1} \mathrm{H}$ NMR (DMSO- $\left.d_{6}\right): \delta$ ppm: 4.49 (bs, H, NH), 7.56-7.60 (m, 2H, nitrophenyl), 7.627.67 (m, 1H, C6-H), 7.72-7.77 (m, 1H, C9-H); 7.87-7.91 (m, $2 \mathrm{H}, \mathrm{C} 7-\mathrm{H}$ and $\mathrm{C} 8-\mathrm{H}), 8.27-8.35$ ( $\mathrm{m}, 2 \mathrm{H}$, nitrophenyl); ${ }^{13} \mathrm{C}$ NMR: , 124.53, 125.45, 126.09, 128.4, 130.64, 131.03, 132.04, $134.23,140.45,142.3,144.0,148.4,161.60$ ( $s p^{2}$ carbons), 171.17 and $181.85(\mathrm{C}=\mathrm{O}), 185.41(\mathrm{C}=\mathrm{S})$; mass $\left(\mathrm{M}^{+}\right)$: 419.40; anal. calcd. (\%) for $\mathrm{C}_{20} \mathrm{H}_{9} \mathrm{~N}_{3} \mathrm{O}_{4} \mathrm{~S}_{2}$ : C, 57.28; H, 2.14; N, 10.02 . found: C, 57.04; H, 1.95; N, 9.84.

11-(4-Chlorophenyl)-2-thioxo-2,3-dihydrotobenzo[g]thiazolo[4,5-b]quinoline-5,10-dione (12c): Green powder, yield: $(56 \%)$; m.p. $>300^{\circ} \mathrm{C}$; IR $\left(\mathrm{KBr}, \mathrm{v}_{\max }, \mathrm{cm}^{-1}\right): 3455(\mathrm{NH})$, 1676 and $1586\left(\mathrm{C}=\mathrm{O}\right.$ of quinone); ${ }^{1} \mathrm{H}$ NMR (DMSO- $\left.d_{6}\right): \delta$ ppm: 4.49 (bs, H, NH), 7.29-7.35 (m, 2H, chlorophenyl), 7.537.58 (m, 2H, chlorophenyl), 7.62-7.67 (m, 1H, C6-H), 7.727.77 (m, 1H, C9-H); 7.87-7.91 (m, 2H, C7-H and $\mathrm{C} 8-\mathrm{H}) ;{ }^{13} \mathrm{C}$ NMR: $124.53,125.45,127.59,128.4,130.64,131.03,132.04$, $134.4,135.02,136.65,140.13,148.4,161.60$ ( $s p^{2}$ carbons), 171.17 and $181.85(\mathrm{C}=\mathrm{O}) 185.41(\mathrm{C}=\mathrm{S})$; mass $\left(\mathrm{M}^{+}\right)$: 408.80; anal. calcd. (\%) for $\mathrm{C}_{20} \mathrm{H}_{9} \mathrm{ClN}_{2} \mathrm{O}_{2} \mathrm{~S}_{2}$ : C, 58.88; H, 2.20; N, 6.86. Found (\%): C, 58.52; H, 1.98; N, 6.55.

11-(4-Methoxyphenyl)-2-thioxo-2,3-dihydrotobenzo [g]thiazolo[4,5-b]quinoline-5,10-dione (12d): Green powder, yield: $(51 \%)$; m.p. $>300{ }^{\circ} \mathrm{C}$; IR $\left(\mathrm{KBr}, \mathrm{v}_{\max }, \mathrm{cm}^{-1}\right): 3433$ and $3343\left(\mathrm{NH}_{2}\right), 1676$ and $1586\left(\mathrm{C}=\mathrm{O}\right.$ of quinone); ${ }^{1} \mathrm{H} \mathrm{NMR}$ (DMSO- $d_{6}$ ): $\delta$ ppm: 3.63 (s, 3H, $\left.\mathrm{OCH}_{3}\right), 4.49$ (bs, H, NH), 7.09-7.15 (m, 2H, methoxyphenyl), 7.43-7.48 (m, 2H, methoxyphenyl), 7.62-7.67 (m, 1H, C6-H), 7.72-7.77 (m, 1H,
C9-H); 7.87-7.91 (m, 2H, C7-H and C8-H); ${ }^{13} \mathrm{C}$ NMR: 56.8 $\left(\mathrm{CH}_{3}\right), 112.40(\mathrm{CN}), 93.6,114.4,124.53,125.45,127.59$, 130.64, 130.95, 131.03, 132.04, 143.0, 146.3, 148.4, 161.60 ( $s p^{2}$ carbons), 171.17 and $181.85(\mathrm{C}=\mathrm{O}) 185.41(\mathrm{C}=\mathrm{S})$; Mass $\left(\mathrm{M}^{+}\right)$: 404.26; anal. calcd. (\%) for $\mathrm{C}_{21} \mathrm{H}_{12} \mathrm{~N}_{2} \mathrm{O}_{3} \mathrm{~S}_{2}$ : C, 62.37; $\mathrm{H}$, 2.97; N, 6.93. Found (\%): C, 62.14; H, 2.69; N, 6.71.

Synthesis of 1-amino-11-aryl-2-mercapto- $1 \mathrm{H}$-benzo[g]imidazo[4,5-b]quinoline-5,10-dione (13a-d): A mixture of compound 11a-d $(0.04 \mathrm{mmol})$ and hydrazine hydrate $(10 \mathrm{~mL}$, $98 \%$ ) was heated under reflux for $3 \mathrm{~h}$. After cooling, water was added and the mixture was neutralized with $10 \%$ hydrochloric acid. The separated crude product was then filtered off and purified by column chromatography using methanol/ chloroform (1:4) as eluent to yield compounds 13a-d.

1-Amino-2-mercapto-11-phenyl-1H-benzo[g]imidazo[4,5-b]quinoline-5,10-dione (13a): Gray powder, yield: (46 $\%)$; m.p. $>300{ }^{\circ} \mathrm{C}$; IR $\left(\mathrm{KBr}, v_{\max }, \mathrm{cm}^{-1}\right)$ : 3320 and $3310\left(\mathrm{NH}_{2}\right)$, $2550(\mathrm{SH}), 1676$ and $1586\left(\mathrm{C}=\mathrm{O}\right.$ of quinone); ${ }^{1} \mathrm{H}$ NMR (DMSO- $d_{6}$ ): $\delta$ ppm: 4.49 (bs, $\left.2 \mathrm{H}, \mathrm{NH}_{2}\right), 7.09-7.18(\mathrm{~m}, 1 \mathrm{H}$, phenyl), 7.27-7.35 (m, 2H, phenyl), 7.46-7.52 ( $\mathrm{m}, 2 \mathrm{H}$, phenyl), 7.62-7.67 (m, 1H, C6-H), 7.72-7.77 (m, 1H, C9-H); 7.87-7.91 (m, 2H, C7-H and C8-H), 12.05 (s, 1H, SH); ${ }^{13} \mathrm{C} \mathrm{NMR:} \mathrm{120.59,}$ 122.40, 124.53, 125.45, 128.4, 130.64, 131.03, 132.04, 133.45, 136.01, 138.7, 143.60, 148.7, 152.6, 153.60 ( $s p^{2}$ carbons), 171.17 and $181.85(\mathrm{C}=\mathrm{O})$; Mass $\left(\mathrm{M}^{+}\right)$: 372.65; anal. calcd. (\%) for $\mathrm{C}_{20} \mathrm{H}_{12} \mathrm{~N}_{4} \mathrm{O}_{2} \mathrm{~S}: \mathrm{C}, 64.51 ; \mathrm{H}, 3.22 ; \mathrm{N}, 15.05$. Found (\%): C, 64.22; H, 3.03; N, 14.89 .

1-Amino-2-mercapto-11-(4-nitrophenyl)-1H-benzo[g]imidazo[4,5-b]quinoline-5,10-dione (13b): Gray powder, yield: $(43 \%)$; m.p. $>300{ }^{\circ} \mathrm{C}$; IR $\left(\mathrm{KBr}, \mathrm{v}_{\max }, \mathrm{cm}^{-1}\right): 3350$ and $3310\left(\mathrm{NH}_{2}\right), 2552(\mathrm{SH}), 1676$ and $1586(\mathrm{C}=\mathrm{O}$ of quinone); ${ }^{1} \mathrm{H}$ NMR (DMSO- $d_{6}$ ): $\delta$ ppm: 4.49 (bs, $2 \mathrm{H}, \mathrm{NH}_{2}$ ), 7.56-7.60 (m, 2H, nitrophenyl), 7.62-7.67 (m, 1H, C6-H), 7.72-7.77 (m, 1H, C9-H); 7.87-7.91 (m, 2H, C7-H and C8-H), 8.27-8.35 (m, 2H, nitrophenyl), 12.05 (s, 1H, SH); ${ }^{13} \mathrm{C}$ NMR: 120.59 , 122.40, 124.53, 125.45, 126.6, 128.4, 130.64, 131.03, 132.04, 136.60, 145.45, 148.7, 152.0, 153.60 ( $s p^{2}$ carbons), 171.17 and $181.85(\mathrm{C}=\mathrm{O})$; Mass $\left(\mathrm{M}^{+}\right)$: 417.56; anal. calcd. $(\%)$ for $\mathrm{C}_{20} \mathrm{H}_{11} \mathrm{~N}_{5} \mathrm{O}_{4} \mathrm{~S}: \mathrm{C}, 57.55 ; \mathrm{H}, 2.63 ; \mathrm{N}, 16.78$. Found (\%): C, 57.21; $\mathrm{H}, 2.63 ; \mathrm{N}, 16.55$.

1-Amino-2-mercapto-11-(4-chlorophenyl)-1H-benzo[g]imidazo[4,5-b]quinoline-5,10-dione (13c): Gray powder, yield: $(48 \%)$; m.p. $>300{ }^{\circ} \mathrm{C}$; IR ( $\left.\mathrm{KBr}, \mathrm{v}_{\max }, \mathrm{cm}^{-1}\right)$ : 3455 and $3437\left(\mathrm{NH}_{2}\right), 2549(\mathrm{SH}), 1676$ and $1586\left(\mathrm{C}=\mathrm{O}\right.$ of quinone); ${ }^{1} \mathrm{H}$ NMR (DMSO- $d_{6}$ ): $\delta$ ppm: 4.49 (bs, $\left.2 \mathrm{H}, \mathrm{NH}_{2}\right), 7.29-7.35$ (m, 2H, chlorophenyl), 7.53-7.58 ( $\mathrm{m}, 2 \mathrm{H}$, chlorophenyl), 7.62-7.67 (m, 1H, C6-H), 7.72-7.77 (m, 1H, C9-H); 7.87-7.91 (m, 2H, C7-H and C8-H), 12.05 (s, 1H, SH); ${ }^{13} \mathrm{C}$ NMR: 122.40, 124.53, 125.45, 128.4, 129.69, 130.64, 131.03, 132.04, 134.4, 136.65, 137.0, 148.7, 152.0, 153.60 ( $s p^{2}$ carbons), 171.17 and 181.85 $(\mathrm{C}=\mathrm{O})$; Mass $\left(\mathrm{M}^{+}\right)$: 406.81; anal. calcd. $(\%)$ for $\mathrm{C}_{20} \mathrm{H}_{11} \mathrm{~N}_{4} \mathrm{O}_{2} \mathrm{SCl}$ : C, 59.11; H, 2.71; N, 13.79. Found (\%): C, 58.92; H, 2.54; N, 13.51 .

1-Amino-2-mercapto-11-(4-methoxyphenyl)-1Hbenzo[g]imidazo[4,5-b]quinoline-5,10-dione (13d): Gray powder, yield: (48\%); m.p. $>300{ }^{\circ} \mathrm{C}$; IR $\left(\mathrm{KBr}, \mathrm{v}_{\max }, \mathrm{cm}^{-1}\right)$ : 3433 and $3393\left(\mathrm{NH}_{2}\right), 2553(\mathrm{SH}), 1676$ and $1586(\mathrm{C}=\mathrm{O}$ of quinone); ${ }^{1} \mathrm{H}$ NMR (DMSO- $\left.d_{6}\right): \delta$ ppm: $3.63\left(\mathrm{~s}, 3 \mathrm{H}, \mathrm{CH}_{3}\right)$, 4.49 (bs, 2H, $\mathrm{NH}_{2}$ ), 7.09-7.15 (m, 2H, methoxyphenyl), 7.43- 
7.48 (m, 2H, methoxyphenyl), 7.62-7.67 (m, 1H, C6-H), 7.727.77 (m, 1H, C9-H); 7.87-7.91 (m, 2H, C7-H and C8-H), 12.05 (s, $1 \mathrm{H}, \mathrm{SH}) ;{ }^{13} \mathrm{C}$ NMR: $56.8\left(\mathrm{CH}_{3}\right), 114.4,124.53,125.45$, $129.19,130.64,130.95,131.03,132.04,153.0,148.7,152.0$, 153.60, 160.14 ( $s p^{2}$ carbons), 171.17 and $181.85(\mathrm{C}=\mathrm{O})$; Mass $\left(\mathrm{M}^{+}\right)$: 402.23; anal. calcd. (\%) for $\mathrm{C}_{21} \mathrm{H}_{14} \mathrm{~N}_{4} \mathrm{O}_{3} \mathrm{~S}: \mathrm{C}, 62.68 ; \mathrm{H}$, 3.48; N, 13.93. Found (\%): C, 62.47; H, 3.26; N, 13.65.

\section{RESULTS AND DISCUSSION}

It is clearly established that 2,3-dichloro-1,4-naphthoquinone (1) reacts with nucleophiles and, depending on their strength, it may undergo substitution of one or both chlorine atoms $^{21}$. Based on the reactivity of $\mathbf{1}$, we have studied its reaction with different phenyl hydrazine, malononitrile, arylmalononitrile and carbon disulphide via undertaking of nucleophilic substitution and Michael addition-elimination reactions.

When 2,3-dichloro-1,4-naphthoquinone (1) was stirred with aryl hydrazine $\mathbf{2 a - d}$ ( 1 equiv.) in ethanol using ice-bath, mono substituted products; 2-chloro-3-(2-arylhydrazinyl)naphthalene-1,4-dione (3a-d) were obtained in a good yield. The reaction of compounds 3a-d with carbon disulphide in dry pyridine was refluxed for 12-16 h to yield 3-arylamino-2-thioxo-2,3dihydro-naphtho[2,3-d]thiazole-4,9-dione (4a-d) (Scheme-I). The latter products were synthesized according to a known method $^{22}$ with minor modification.

The spectroscopic analysis using IR, NMR and MS conformed the structure of compounds 4a-d. For instance, IR spectra of compound $\mathbf{4 a}$ revealed the presence of vibration bands for $\mathrm{NH}$ at 3300 and $3250 \mathrm{~cm}^{-1}$, carbonyl groups at 1672 and $1596 \mathrm{~cm}^{-1}$, as well as thione group at $1190 \mathrm{~cm}^{-1}$. Moreover, ${ }^{13} \mathrm{C}$ NMR spectrum for compound $\mathbf{4 a}$, showed the signals of both $\mathrm{C}=\mathrm{O}$ groups at $\delta 171$ and $181 \mathrm{ppm}$, while that for $\mathrm{C}=\mathrm{S}$ appeared at $\delta 184 \mathrm{ppm}$.

We also conducted a reaction of 2,3-dichloronaphthoquinone 1 with malonitrile or arylmalononitrile in the presence of ammonium acetate. The generated precursor is then used for the preparation of naphthoquinones containing indole, quinoline, thiazole and imidazole fragments.

In this context, reaction of naphthoquinone $\mathbf{1}$ with malonitrile and ammonium acetate in ethanol afforded 2-amino4,9-dioxo-4,9-dihydro-1H-benzo[f]indole-3-carbonitrile (5) (Scheme-II). The method used for the synthesis of compound $\mathbf{5}$ is shown in Scheme-II. Furthermore, we have adopted one-pot multicomponent reactions for 2-amino-4,9-dioxo-4,9dihydro- $1 H$-benzo[f]indole-3-carbonitrile synthesis. The outcome of this synthetic approach is significant when noting that Ryu et al. ${ }^{23}$ have prepared the structurally and conceptually related 2-amino-1-alkyl and aryl-4,9-dioxo-4,9-dihydro- $1 H$ benzo[f]indole-3-carbonitriles in two steps.

The Hantzsch-type reaction, performed in this study, furnished 2-amino-3-cyano-4,9-dihydro- $1 \mathrm{H}$-benzo[f]indole4,9-dione in very good yields, in spite of the number of steps involved. This reaction was carried out under metal-free conditions and in the presence of ammonium acetate, a soft Bronsted acid, which served as a reactant and a catalyst as well. The catalytic role of ammonium acetate in this reaction is evident by noting that the reaction did not take place when ammonia is used instead of the ammonium acetate.

We have also synthesized 2-amino-4,9-dioxo-4,9-dihydro$1 H$-benzo[f]indole-3-carbonitrile 5 by nucleophilic substitution of both $\mathrm{Cl}$ using Michael addition-elimination reactions, in the presence of activated methylene group and ammonia solution.

Description of the method employed for the synthesis of potassium 3-cyano-4,9-dioxo-4,9-dihydro- $1 H$-benzo[f]indol2-ylcarbamodithioate (6) is depicted in Scheme-II. The synthesis was performed following the method developed by El-Emam et al. ${ }^{24}$, where the reaction of 2-amino-4,9-dioxo-4,9-dihydro$1 \mathrm{H}$-benzo[f]indole-3-carbonitrile (5) is allowed to react with carbon disulphide in ethanolic potassium hydroxide solution. The resultant product is identified as potassium 3-cyano-4,9dioxo-4,9-dihydro- $1 H$-benzo[f]indol-2-ylcarbamodithioate (6). Additionally, the cyanide group in $\mathbf{6}$ was hydrolyzed by concentrated sulphuric acid to the corresponding carboxylic acid $(\mathrm{COOH})$ group. Nucleophilic substitution of the $\mathrm{COOH}$ group by $\mathrm{SH}$ yielded the new target compound 2-thioxo- $2 \mathrm{H}$ benzo[f]thiazolo[4,5-b]indole-5-10(3H,4H)-dione (7). Compound 7 was also prepared by intramolecular cyclization of compound 6 in the presence conc. $\mathrm{H}_{2} \mathrm{SO}_{4}$, by adopting the same procedure reported as in literature ${ }^{24,25}$ followed by purification by column chromatography to produce a green solid in $60 \%$ yield.

Spectral characterization of compound 7 via IR, ${ }^{1} \mathrm{H}$ NMR and ${ }^{13} \mathrm{C}$ NMR confirmed its existence in the thione form. Careful inspection of the IR spectra of this compound showed the

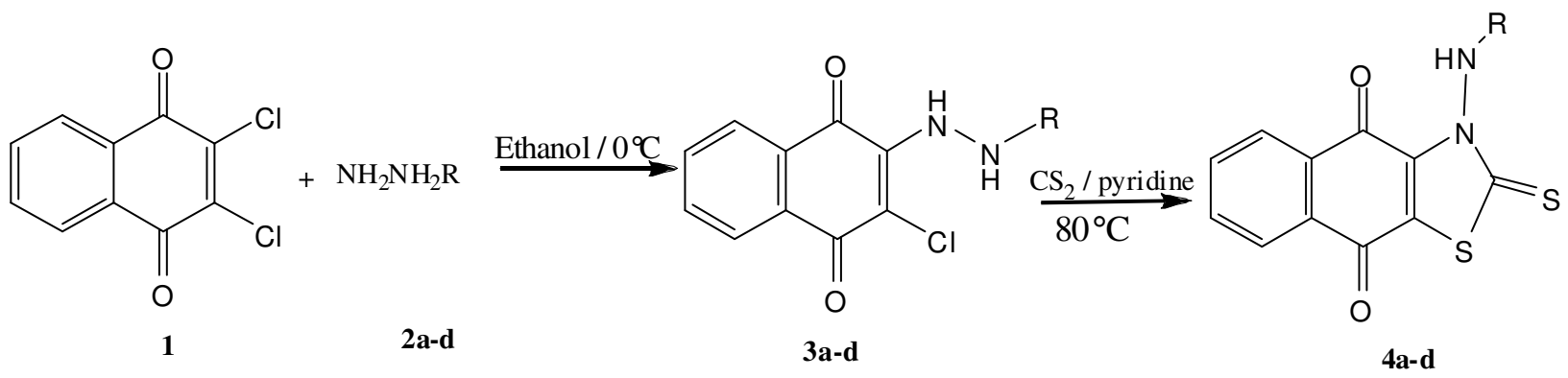

(a) $\mathrm{R}=\mathrm{C}_{6} \mathrm{H}_{5}$

(b) $\mathrm{R}=4-\mathrm{NO}_{2} \mathrm{C}_{6} \mathrm{H}_{4}$

(c) $\mathrm{R}=4-\mathrm{ClC}_{6} \mathrm{H}_{4}$

(d) $\mathrm{R}=4-\mathrm{CH}_{3} \mathrm{OC}_{6} \mathrm{H}_{4}$ 


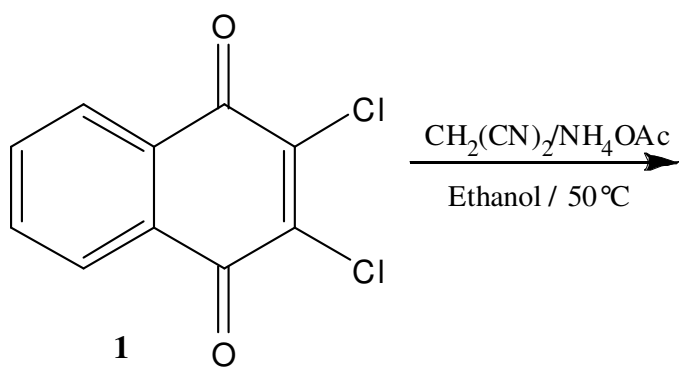<smiles>CCOCCOCCOCC</smiles><smiles>O=C1c2ccccc2C(=O)c2c1[nH]c1[nH]c(=S)sc21</smiles><smiles>O=C(O)CCCCCCO</smiles><smiles>[Y][Sb]C(=S)Nc1[nH]c2c(c1C#N)C(=O)c1ccccc1C2=O</smiles><smiles></smiles>

Scheme-II

absence of cyanide peak and the presence of the common characteristic absorption peaks at $3428 \mathrm{~cm}^{-1}$ for $(\mathrm{NH})$, as well as 1656 and $1586 \mathrm{~cm}^{-1}$ for $\left(\mathrm{C}=\mathrm{O}\right.$ of quinone). The ${ }^{1} \mathrm{H}$ NMR spectrum displayed the nitrogen proton as singlet at $\delta 4.43(1 \mathrm{H})$ and the aromatic protons as different multiples from $\delta 7$ to $7.91 \mathrm{ppm}$. In addition, the ${ }^{13} \mathrm{C}$ NMR spectrum showed the $s p^{2}$ carbons at $\delta 121.40,125.34,126.27,131.43,131.85,132.83$, 134.26 and 164.35 , the $\mathrm{C}=\mathrm{O}$ at $\delta 171.97$ and 182.645 and the $\mathrm{C}=\mathrm{S}$ at $\delta 184.52 \mathrm{ppm}$.

On the other hand, when potassium 3-cyano-4,9-dioxo4,9-dihydro- $1 H$-benzo[f]indol-2-ylcarbamodithioate (6) was heated with hydrazine hydrate, it gave rise to 1-amino-2mercaptobenzo[f]imidazo[4,5-b]indole-5-10 $(1 H, 4 H)$-dione (8) as the major product.

The structure of compound $\mathbf{8}$ was predicted on the basis of its IR, ${ }^{1} \mathrm{H}$ NMR, ${ }^{13} \mathrm{C}$ NMR and mass spectral data. The IR spectra of this compound showed the characteristic absorption bands for (NH) at $3328 \mathrm{~cm}^{-1}, \mathrm{NH}_{2}$ at 3155 and $3033 \mathrm{~cm}^{-1}$, SH at $2560 \mathrm{~cm}^{-1}$, as well as quinone $\mathrm{C}=\mathrm{O}$ at 1656 and $1586 \mathrm{~cm}^{-1}$. Moreover, the ${ }^{1} \mathrm{H}$ NMR spectra of the compound revealed the presence of the nitrogen protons as two singlet at $\delta 4.43$ and 6.66 and the aromatic carbons as a different multiples from $\delta$ 7 to 7.91 , while the SH proton appears as a singlet at $\delta 12.05$ ppm. The ${ }^{13} \mathrm{C}$ NMR spectra for compound $\mathbf{8}$ gave evidence for the aryl and diazole carbon atoms at $\delta 121.40,125.34,126.27$, $131.43,131.85,132.83,134.26$ and 164.35 along with the $\mathrm{C}=\mathrm{O}$ peaks at $\delta 171.97$ and $182.64 \mathrm{ppm}$.

One-pot multicomponent reactions have been also adopted for the synthesis of the 2-amino-4-aryl-5,10-dioxo-5,10dihydrobenzo $[g]$ quinoline-3-carbonitrile (10a-d), following the reflux method for a mixture 2-arylidenemalononitrile (9a-d) and 2,3-dichloro-1,4-naphthoquinone $\mathbf{1}$ in the presence of ammonium acetate (Scheme-III). The structures of synthesized compounds 10a-d were confirmed on the basis of their spectroscopic results.

After structural characterizations the compounds 10a-d were allowed to react at room temperature with carbon disulphide in the presence of potassium hydroxide in ethanol, to generate the corresponding potassium 4-aryl-3-cyano-5,10dioxo-5,10-dihydrobenzo[g]quinolin-2-yl-carbamodithiate (11a-d) compounds. Importantly, cyclization of the resultant products (11a-d) by using concentrated sulphuric acid, at room temperature yielded the new products 11-aryl-2-thioxo-2,3dihydrotobenzo[g]thiazolo[4,5-b]quinoline-5,10-dione (12a-d) as illustrated in Scheme-III. Formation of compounds 12a-d is probably occurs by acid hydrolysis of the $\mathrm{CN}$ group in the 
<smiles>N#CC(C#N)=CC[18O]</smiles><smiles>Cc1c2c(nc3[nH]c(=S)sc13)C(=O)c1ccccc1C2=O</smiles>

12a-d

(a) $\mathrm{R}=\mathrm{C}_{6} \mathrm{H}_{5}$

(b) $\mathrm{R}=4-\mathrm{NO}_{2} \mathrm{C}_{6} \mathrm{H}_{4}$

(c) $\mathrm{R}=4-\mathrm{ClC}_{6} \mathrm{H}_{4}$

(d) $\mathrm{R}=4-\mathrm{CH}_{3} \mathrm{OC}_{6} \mathrm{H}_{4}$<smiles>N#Cc1cc2c(nc1NC(=S)[SeH])C(=O)c1ccccc1C2=O</smiles>

11a-d $50{ }^{\circ} \mathrm{C}$

\section{Scheme-III}<smiles>[R]c1c2c(nc3nc(S)n(N)c13)C(=O)c1ccccc1C2=O</smiles>

13a-d

precursors potassium 4-aryl-3-cyano-5,10-dioxo-5,10dihydrobenzo $[g]$ quinolin-2-ylcarbamodithiate11a-d to yield the carboxylic acid $(\mathrm{COOH})$ derivatives. The final products (12a-d) were accomplished by nucleophilic substitution of $\mathrm{SH}$ on the $\mathrm{COOH}$ group followed by intramolecular cyclization.

Careful analysis of the spectral data obtained for compounds 12a-d disclosed their existences as thione forms. For instance the IR spectra of the compound 12a showed the presence of the characteristic absorption bands at $3350 \mathrm{~cm}^{-1}(\mathrm{NH}), 1656$ and $1586 \mathrm{~cm}^{-1}\left(\mathrm{C}=\mathrm{O}\right.$ of quinone). The ${ }^{1} \mathrm{H}$ NMR spectrum showed the nitrogen proton as singlet at $\delta 4.49(1 \mathrm{H})$ and the aromatic protons as different multiples from $\delta 7.09$ to 7.91 ppm. The ${ }^{13} \mathrm{C}$ NMR spectrum attested for the presence of the $s p^{2}$ carbons at $\delta 119.40,120.59,124.53,125.45,128.4,130.64$, $131.03,132.04,133.45,143.60,148.7,150.6,161.60 \mathrm{ppm}$, the two $\mathrm{C}=\mathrm{O}$ at $\delta 171.17$ and $181.85 \mathrm{ppm}$ and the $\mathrm{C}=\mathrm{S}$ at $\delta$ $185.41 \mathrm{ppm}$.

Moreover, potassium 4-aryl-3-cyano-5,10-dioxo-5,10dihydrobenzo[g]quinolin-2-ylcarbamodithiate compounds (11a-d) were heated with hydrazine hydrate, very good yields of 1-amino-11-aryl-2-mercapto-1H-benzo[g]imidazo[4,5b]quinoline-5,10-dione derivatives (13a-d) were obtained. Similarly, the proposed mechanism for formation of compounds 13a-d involves initially nucleophilic substitution of $\mathrm{S}$ by $\mathrm{NH}_{2}$ followed by another nucleophilic substitution of $\mathrm{CN}$ by $\mathrm{NH}$.

Consistent with other derivatives, spectral data obtained for compounds 13a-d confirmed their existence in the thiol form. This is clearly evidenced via IR data of the parent compound 13a, which revealed the presence of the vibration bands of $\mathrm{NH}_{2}$ at 3320 and $3310 \mathrm{~cm}^{-1}$, along with a carbonyl group at 1676 and $1586 \mathrm{~cm}^{-1}$. Additionally, the ${ }^{1} \mathrm{H}$ NMR results are consistent with the presence of nitrogen protons as a singlet at $\delta 4.49$ (bs, $2 \mathrm{H}, \mathrm{NH}_{2}$ ) and the aromatic protons as different multiples from $\delta 7.09$ to 7.91 , whereas, the SH proton appeared as a singlet at $\delta 12.05 \mathrm{ppm}$. The ${ }^{13} \mathrm{C}$ NMR data also lend additional support concerning identification of 13a structure. The obtained spectrum showed the presence of the aryl and the diazole carbons at $\delta 120.59,122.40,124.53,125.45,128.4$, 
130.64, 131.03, 132.04, 133.45, 136.01, 138.7, 143.60, 148.7, 152.6 , respectively, together with a $153.60 \mathrm{ppm}$ peak for $s p^{2}$ carbons the characteristic peaks for $\mathrm{C}=\mathrm{O}$ are detected at 171.17 and $181.85 \mathrm{ppm}$.

\section{Conclusion}

In the present work, we reported one-pot multicomponent synthesis of 2-amino-4,9-dioxo-4,9-dihydro- $1 \mathrm{H}$-benzo[f]indole-3-carbonitrile (5) and 2-amino-4-aryl-5,10-dioxo-5,10dihydrobenzo[g] quinoline-3-carbonitrile (10a-d). We have also demonstrated the feasibility of the the Michael additionelimination reaction of compounds $\mathbf{3 a - d}, \mathbf{5}$ and $\mathbf{1 0 a}-\mathbf{d}$ with carbone disulphide in presence of pyridine or conc. $\mathrm{H}_{2} \mathrm{SO}_{4}$ or hydrazine hydrate gives respectively the compounds 3arylamino-2-thioxo-2,3-dihydro-naphto[2,3-d]thiazole-4,9dione (4a-d), 2-thioxo-2H-benzo[f]thiazolo[4,5-b]indole-510(3H,4H)-dione (7), 2-Mercaptobenzo[f]thiazolo[4,5b]indole-5-10(1H,4H)-dione (8), 11-aryl-2-thioxo-2,3-dihydrotobenzo[g]thiazolo[4,5- $b$ ]quinoline-5,10-dione (12a-d) and 1-amino-11-aryl-2-mercapto- $1 H$-benzo[ $g$ ]imidazo[4,5$b$ ]quinoline-5,10-dione (13a-d). Structural elucidation of the target compounds was fully demonstrated by various spectral data.

The biological activities concerning the antibacterial and antifungal of all products prepared in this work are under investigations.

\section{ACKNOWLEDGEMENTS}

The authors extended their sincere appreciation to the Deanship of Scientific at King Saud University for its funding of this research through the Research Group Project No. RGPVPP-222.

\section{REFERENCES}

1. S. Spyroudis, Molecules, 5, 1291 (2000)

2. M. Tisler, In ed.: A.R. Katritzky, Advances in Heterocyclic Chemistry; Heterocyclic Quinones; Academic: London, Vol. 45, p. 37 (1989).
3. M.B. Teimouria and H.R. Khavasi, Tetrahedron, 63, 10269 (2007).

4. H. Roberts, W.M. Choo, S.C. Smith, S. Mrzuki, A.W. Linnane, T.H. Porter and K. Folkers, Arch. Biochem. Biophys., 191, 306 (1978).

5. J.P. di Rigo, C. Bruel, L.A. Graham, P. Sonimski and B.L. Trumpower, J. Biol. Chem., 271, 15341 (1996).

6. R. Chung-Kyu, Y.L. Jung, H.J. Seong and N. Ji-Hee, Bioorg. Med. Chem. Lett., 19, 146 (2009).

7. S. Ganapaty, P.S. Thomas, G. Karagianis, P.G. Waterman and R. Brun, Phytochemistry, 67, 1950 (2006).

8. T.M.S. Silva, C.S. Camara, T.P. Barbosa, A.Z. Soares, L.C. da Cunha, A.C. Pinto and M.D. Vargas, Bioorg. Med. Chem., 13, 193 (2005).

9. C. Biot, H. Bauer, R.H. Schirmer and E. Davioud-Charret, J. Med. Chem., 47, 5972 (2004).

10. A. Mantyla, T. Garnier, J. Rautio, T. Nevalainen, J. Vepsalainen, A. Koskinen, S.L. Croft and T. Jarvinen, J. Med. Chem., 47, 188 (2004).

11. V.K. Tandon, H.K. Maurya, A. Tripathi, G.B. ShivaKesva, P.K. Shukla, A. Srivastava and D. Panda, Eur. J. Med. Chem., 44, 1086 (2009).

12. V.K. Tandon, H.K. Maurya, D.B. Yadav, A. Tripathi, M. Kumar and P.K. Shukla, Bioorg. Med. Chem. Lett., 16, 5883 (2006).

13. G. Tudor, P. Gutierrez, A. Aguilera-Gutierrez and E.A. Sausville, Biochem. Pharmacol., 65, 1061 (2003).

14. C.-K. Ryu, S.-Y. Lee, N.Y. Kim, J.A. Hong, J.H. Yoon and A. Kim, Bioorg. Med. Chem. Lett., 21, 427 (2011).

15. C.-K. Ryu, R.-E. Park, M.-Y. Ma and J.-H. Nho, Bioorg. Med. Chem. Lett., 17, 2577 (2007).

16. C.A. Camara, A.C. Pinto, M.A. Rosa and M.D. Vargas, Tetrahedron, 57, 9569 (2001).

17. H. Becker, Organicum (Practical Handbook of Organic Chemistry). Addison-Wesley, MA, USA, p. 611 (1973).

18. F.E.M. El-Baih, H.H. Al-Rasheed and H.M. Al-Hazimi, J. Saudi Chem. Soc., 9, 575 (2006).

19. J.B. Lambert and E.P. Mazzola, NMR Spectroscopy, An Introduction to Principles, Applications and Experimental Methods, Pearson Education Inc., New Jersey (2004).

20. M.L. Deb and P.J. Bhuyan, Tetrahedron Lett., 46, 6453 (2005).

21. K.T. Finley, In ed.: S. Patai, The Chemistry of Quinonoid Compounds; John Wiley \& Sons, Vol. 2, pp. 877-1144 (1974).

22. H.N. Hafez, H.A.R. Hussein and A.B.A. El-Gazzar, Eur. J. Med. Chem., 45, 4026 (2010).

23. C.-K. Ryu, J.Y. Lee, S.H. Jeong and J.-H. Nho, Bioorg. Med. Chem. Lett., 19, 146 (2009).

24. A.A. El-Emam, A. Moustafa, A.M. Abdelal and M.B. El-Ashmawy, Chin. Pharm. J., 45, 101 (1993).

25. F.E.M. El-Baih, Sh. I. Al-Aqeel, O.A. El-Sayed and H.M. Al-Hazimi, J. Saudi Chem. Soc., 18, 1 (2005). 